\title{
Opposing Influences of Affective State Valence on Visual Cortical Encoding
}

\author{
Taylor W. Schmitz, ${ }^{1,2}$ Eve De Rosa, ${ }^{1,2,3}$ and Adam K. Anderson ${ }^{1,2,3}$ \\ ${ }^{1}$ Department of Psychology, ${ }^{2}$ University of Toronto Neuroscience Program, and ${ }^{3}$ Rotman Research Institute, University of Toronto, Toronto, Ontario M5S \\ 3G3, Canada
}

\begin{abstract}
Positive and negative emotional states are thought to have originated from fundamentally opposing approach and avoidance behaviors. Furthermore, affective valence has been hypothesized to exert opposing biases in cognitive control. Here we examined with functional magnetic resonance imaging whether the opposing influences of positive and negative states extend to perceptual encoding in the visual cortices. Based on prior behavioral research, we hypothesized that positive states would broaden and negative states would narrow visual field of view (FOV). Positive, neutral, and negative states were induced on alternating blocks. To index FOV, observers then viewed brief presentations $(300 \mathrm{~ms})$ of face/place concentric center/surround stimuli on interleaved blocks. Central faces were attended, rendering the place surrounds unattended. As face and place information was presented at different visual eccentricities, our physiological metric of FOV was a valence-dependent modulation of place processing in the parahippocampal place area (PPA). Consistent with our hypotheses, positive affective states increased and negative states decreased PPA response to novel places as well as adaptation to repeated places. Individual differences in self-reported positive and negative affect correlated inversely with PPA encoding of peripheral places, as well as with activation in the mesocortical prefrontal cortex and amygdala. Psychophysiological interaction analyses further demonstrated that valence-dependent responses in the PPA arose from opponent coupling with extrafoveal regions of the primary visual cortex during positive and negative states. These findings collectively suggest that affective valence differentially biases gating of early visual inputs, fundamentally altering the scope of perceptual encoding.
\end{abstract}

\section{Introduction}

Emotionally significant stimuli receive privileged neural processing (Pessoa and Ungerleider, 2004; Anderson, 2005; Vuilleumier, 2005). Corticosubcortical prefrontal and amygdalar pathways are thought to influence, or in some cases bypass, top-down attentional control to facilitate appraisals of and responses to affective information (Cohen et al., 2007; Dolan, 2007). This neural prioritization of emotionally significant information appears to reflect motivational importance (i.e., high vs low) rather than motivational direction (i.e., approach vs avoid). Extant neuroimaging evidence suggests a common effect of physiological arousal on perceptual cortical processing, with greater overall magnitude of visual cortical response to both positive and negative stimuli (Lang et al., 1998; Bradley et al., 2003).

In contrast, behavioral research suggests a critical distinction between the perceptual correlates of positive and negative affective states (Derryberry and Tucker, 1994; Rowe et al., 2007), with positive affect broadening and negative affect narrowing the distribution, or scope, of one's field of view (FOV). For example, when observers are asked to selectively attend to a central target

Received Nov. 7, 2008; revised April 23, 2009; accepted April 27, 2009.

This research was supported by the Canadian Institutes of Health Research (E.D.R.) and the National Science and Engineering Research Council (A.K.A.). We thank Matt Dixon for assistance with data collection and Josh Susskind for assistance with stimulus preparation.

Correspondence should be addressed to Taylor W. Schmitz, Department of Psychology, University of Toronto, 100 St. George Street, Toronto, 0N M5S 3G3, Canada. E-mail: taylor@aclab.ca.

D01:10.1523/JNEUROSCI.5387-08.2009

Copyright $\odot 2009$ Society for Neuroscience $\quad$ 0270-6474/09/297199-09\$15.00/0 and ignore irrelevant flanking distractors, induction of positive affect results in greater flanker interference than negative affect (Fenske and Eastwood, 2003; Rowe et al., 2007). Similarly, positive mood states are associated with a greater tendency to perceive global components of visuospatial stimuli, whereas participants in negative moods tend to perceive local components (Gasper and Clore, 2002). The narrowing of FOV by negative affect is sometimes referred to as "weapon focus," where a focal stimulus component is encoded at the expense of peripheral details, as measured in later memory (Christianson, 1992; Kensinger et al., 2007a).

However suggestive, these behavioral data may originate in higher-level cognitive control biases rather than altered perceptual encoding. We hypothesized that if the corticosubcortical pathways underlying positive and negative states do differentially influence FOV, then activation of these systems would yield opposing influences on cortical encoding of unattended extrafoveal information. Using functional magnetic resonance imaging (fMRI), observers viewed alternating epochs of emotionally laden images (positive, neutral, and negative valence) and performed a neutral interleaved visuospatial task (VS) (Fig. 1). In contrast to prior neuroimaging studies, we assessed not only cortical/subcortical response to emotion-evoking images, but also how engagement of these systems alters information processing in a discrete visuospatial task. We were therefore able to dissociate the neural effects of endogenous affective states from exogenous affective stimuli.

In the visuospatial task, observers attended to a neutral face 
briefly presented at the foveal center with an unattended place in the peripheral surround. The visuospatial task capitalized on object-sensitive cortical processing of attended faces in the extrastriate fusiform face area (FFA) and unattended places in the parahippocampal place area (PPA). Previous studies have found that increasing perceptual load of a foveal target task decreases cortical encoding of ignored peripheral stimuli (Yi et al., 2004). Specifically, PPA adaptation to repeated peripheral places, as measured by repetition reduction in blood oxygenation leveldependent (BOLD) response, is abolished during inattention. As such, the place surround was repeated within our visuospatial task, thereby providing a more precise index of peripheral stimulus encoding (Levy et al., 2001; Yi et al., 2004). Hence, our physiological metrics of opposing biases on FOV was a valence-dependent change in the magnitude of PPA response to both novel places and repeated places.

\section{Materials and Methods \\ Procedures \\ Participants}

Sixteen healthy volunteers with normal vision ( 9 females; mean age $=22.03, \mathrm{SD}=0.68$ ) participated in this Institutional Review Board approved study. Written informed consent was obtained before study procedures. Two participants were removed from subsequent fMRI analyses, one due to scanner malfunction and the other due to unreported atypical vision.

\section{Face/place localizer task}

The PPA and FFA were localized using a block-design task consisting of alternating epochs of face and place stimuli (supplemental Methods, available at www.jneurosci.org as supplemental material). Participants made gender decisions in the face blocks and interior/exterior decisions in the house blocks. The stimuli were presented serially for $4000 \mathrm{~ms}$ in trains of like events that varied pseudorandomly from four to six trials (equated between conditions), with six epochs of each condition in total. This variation reduced condition predictability while preserving efficiency of the boxcar stimulus function.

\section{Experimental task}

The block design experiment was composed of alternating blocks of affect induction (AI) and interleaved blocks of the VS (Fig. 1). To generate sustained affective states, the three AI conditions (positive, neutral, or negative) were arranged sequentially in groups of four AI-VS cycles of like valence. Each AI condition was collected once per scan session, across two scan sessions (for a total of six discrete valence inductions). The order of the AI conditions was inverted for each scan session to counterbalance (within subjects) potential carryover effects from the positive and negative conditions. The session order was also counterbalanced between subjects.

In the AI blocks, 10 International Affective Picture System (IAPS) images were serially presented for $2000 \mathrm{~ms}$ each. Participants passively viewed the images. We used sparse sampling of affective states to prevent suppression of emotional response by cognitive labeling (Goel and Dolan, 2003). As such, intrascan measures of the mood manipulation were collected only after the fourth and final AI-VS cycle of each valence induction. Our manipulation check of sustained affect induction was therefore determined from self-report measures acquired once per va- lence induction (and subsequent to the last VS block). Participants were prompted to rate their self-perceived valence and arousal, respectively, on separate Likert scales derived from the IAPS: 1 (negative valence/low arousal) to 9 (positive valence/high arousal) (Lang et al., 1999).

In the VS blocks, 10 face/house compound stimuli were presented serially (supplemental Methods, available at www.jneurosci.org as supplemental material), each for $300 \mathrm{~ms}$, with a $1700 \mathrm{~ms}$ interstimulus interval. Because human perceptual acuity declines with increasing eccentricity in the visual field (Weymouth, 1958; Rovamo et al., 1978), houses were scaled in size relative to the faces to equate the accuracy of foveal and extrafoveal processing. A cortical magnification factor for the extrafoveal house information was derived from neuroimaging studies of the eccentricity bias of similar face/house composite stimuli (Levy et al., 2001, 2004). To approximate isoacuity, the face component subtended a visual angle of $2.2^{\circ}$, whereas the house component subtended $14.3^{\circ}$ (at $117 \mathrm{~cm}$ viewing distance).

Short stimulus durations were used to maximize attentional engagement to the central face task and minimize overt and covert shifts of attention to the periphery. Participants were instructed to determine the gender of the face component and maintain central fixation throughout the trial. A fixation cue composed of a circular border and a central fixation point replaced the faces during stimulus offsets. The central fixation point was displayed continuously throughout trials to maintain central gaze. Male and female faces were distributed equally but varied pseudorandomly within blocks. The attended face stimuli did not repeat at any point throughout the experiment. Within each block, 5 novel house flankers were serially presented [exposure $1(\mathrm{e} 1)$ ] and then repeated in the same order [exposure 2 (e2)]. Between blocks of the VS task, place images did not repeat at any point throughout the experiment.

To provide a more complete assessment of affect induction and to acquire self-report for each of the associated AI blocks, immediately following the scan, an additional measure of the valence manipulation 
was obtained. Participants were prompted after each 10-trial AI block to report both their self-perceived valence and arousal. Condition order was randomized by block. Intrascan and postscan ratings of valence and arousal (mean values) were examined with Pearson correlations to assess whether postscan ratings were a reliable indicator of the sparser sampling of intrascan affective states. Good internal reliability was observed for both ratings of valence $(r=0.78, p<0.001)$ and arousal $(r=0.78, p<$ $0.001)$. As intrascan ratings were acquired only once per valence induction, we used the extrascan self-reports as our index of AI because it afforded a larger sample of data points per subject.

\section{Setup}

Imaging data were collected with a Siemens Allegra 3.0 T scanner operating at a slew rate of $400 \mathrm{~T} / \mathrm{m} / \mathrm{s}$ and a 12-channel asymmetric gradient head coil. The block design experiment was designed and implemented using the software package Presentation (version 9.81; NeuroBehavioral Systems). Before scanning, participants were provided with instruction and practice on the fMRI task. Low-amplitude transistor-transistor logic pulses were monitored via a parallel port cable to synchronize slice acquisition and stimulus delivery with submillisecond accuracy. Stimuli were presented on a rear-mounted projection screen, set at a (native) $1024 \times 768$ resolution.

\section{Structural imaging}

For each subject, a three-dimensional magnetization-prepared rapidacquisition gradient echo pulse sequence was used to obtain a highresolution T1-weighted structural volume. The imaging parameters were as follows: repetition time $(\mathrm{TR})=2000 \mathrm{~ms}$; echo time $(\mathrm{TE})=2.63 \mathrm{~ms}$; matrix $=256 \times 160 ;$ FOV $=256 \times 256$; slice thickness $=1 \mathrm{~mm} ; 160$ axial oblique slices; total acquisition time $=6.5 \mathrm{~min}$.

\section{Echo-planar imaging}

For each subject, a T2*-weighted gradient-echo echo-planar image (EPI) pulse sequence was prescribed and higher order shimmed for the functional trials. The EPI parameters were as follows: $\mathrm{TE}=30 \mathrm{~ms}$; $\mathrm{TR}=2000$ $\mathrm{ms}$; flip angle $=270^{\circ}$; acquisition matrix $=64 \times 64 ; \mathrm{FOV}=200 \mathrm{~mm}$. Thirty axial oblique slices of the brain were acquired at each time point, with a voxel resolution of $3.1 \times 3.1 \times 5 \mathrm{~mm}$, no skip between slices. For the localizer task, 153 time points were collected, of which the first 12 were discarded (for a total of 141 reconstructed time points). In each of the two experimental task runs, 319 time points were collected, of which the first 11 and last two were discarded (for a total of 612 reconstructed time points).

\section{Data analysis \\ Preprocessing}

Functional activation was determined from the BOLD signal using the software Statistical Parametric Mapping (SPM5, University College London, London, UK; http://www.fil.ion.ucl.ac.uk/spm/software/spm5). Following image reconstruction (SPM5 DICOM import utility), the time series data for each participant were motion corrected (translational motion parameters were less than one voxel for all included participants) and coregistered with their T1-weighted structural image. The T1 image was bias corrected and segmented using template (International Consortium for Brain Mapping) tissue probability maps for gray/white matter and CSF. Warping parameters were obtained from the tissue segmentation procedure and subsequently applied to the time series data (resampling to $3 \mathrm{~mm}^{3}$ voxels). The time series data were spatially smoothed to a $6 \mathrm{~mm}^{3}$ full-width half maximum Gaussian kernel. Last, a voxel-level detrending procedure was applied to remove time series components correlated with global fluctuations in the BOLD signal (Macey et al., 2004).

\section{First-level statistical models}

Single subject time series data were submitted to first-level general linear statistical models (Friston et al., 1994) examining the face/place localizer and experimental task, respectively. Using the SPM5 design specification, the task-specific boxcar stimulus functions were convolved with the canonical hemodynamic response function (HRF). Each model included within-session global scaling (default), high-pass filtering to remove low- frequency signal drift (period $=128 \mathrm{~s}$ ), and the AR1 method of estimating temporal autocorrelation.

For the face/place localizer, two condition-specific regressors were included modeling face and place blocks. Separate $t$ contrasts were specified for [places $>$ faces] and [places $<$ faces]. For the experimental task, nine condition-specific regressors were included in the first-level model. Columnwise, these regressors modeled the three AI conditions (positive, neutral, negative) and six for the VS conditions (valence $\times$ repetition). Positive effect $t$ contrasts were generated for each regressor column.

\section{Second-level statistical models}

ROI analyses. Because face and place information was presented simultaneously and at different visual eccentricities during the VS task, we performed conjunction analyses to determine areas of functional convergence between the separate object localizers (face and place) and the VS conditions. To calculate the [localizer and VS] conjunction for place and face processing, we submitted a set of $t$ contrasts corresponding to the respective place and face localizer and a set of $t$ contrasts corresponding to el conditions of the VS task to second-level repeated-measures ANOVAs. Significant conjunction effects (set at a conjoint $\alpha$ of $p_{\text {unc }}<0.01$ ) were found in the FFA and PPA, indicating common object-sensitive activation at the voxel level between localizer and VS tasks (Fig. 2a). The place conjunction localized bilateral clusters extending along the collateral sulcus just ventral to the posterior hippocampus, consistent with the parahippocampal place area (Aguirre et al., 1998; Epstein and Kanwisher, 1998; Levy et al., 2004). Center-of-mass locations [Montreal Neurological Institute (MNI) space] and volume for the PPA ROIs are as follows: left PPA, $x=-22, y=-45, z=-10\left(8397 \mathrm{~mm}^{3}\right)$; right PPA, $x=24, y=$ $-44, z=-10\left(10,881 \mathrm{~mm}^{3}\right)$. The face conjunction localized clusters bilaterally in the lateral aspect of the posterior fusiform gyrus, consistent with the fusiform face area (Puce et al., 1995; Kanwisher et al., 1997). Center-of-mass locations (MNI space) and volume for the FFA ROIs are as follows: left FFA, $x=-37, y=-52, z=-23\left(135 \mathrm{~mm}^{3}\right)$; right FFA, $x=40, y=-49, z=-20\left(756 \mathrm{~mm}^{3}\right)$.

The resultant FFA and PPA ROIs were used as functionally defined masks to interrogate main and interaction effects for a 3 (valence) $\times 2$ (repetition) repeated-measures factorial ANOVA modeling all conditions of the VS task. Main and interaction $(F)$ effects for the ROI analyses were interrogated using an uncorrected $p<0.01$ height and a 5 voxel extent as base thresholds. Voxels surviving a small volume correction using the false discovery rate (Genovese et al., 2002) set at $p<0.05$ were deemed statistically significant. To further interrogate factorial effects in these clusters, percentage signal change was extracted in the first-level models for each condition ( $\beta$ coefficient) against implicit baseline (i.e., $\beta$ coefficient of the session constants) using Marsbar software (http:// marsbar.sourceforge.net/) (Brett et al., 2002).

Brain-behavior analyses. We examined whether continuous behavioral measures of self-reported valence (rating acquired from the affect induction) were correlated with corresponding indices of percentage signal change in the extrastriate ROIs. Linear regression models were used to evaluate the brain-behavior relationship. We examined the relationship between behavioral ratings of valence and the magnitude of signal change to novel places (e1) and magnitude of repetition reduction (e2 minus e1) blocks within each valence condition (with larger magnitude indicated by greater negative differences).

Psychophysiological interaction analyses. The significant valence $\times$ repetition interactions within the left $(x=-30, y=-45, z=-15)$ and right ( $x=36, y=-30, z=-18)$ PPA were subsequently used as seeds for two respective psychophysiological interaction (PPI) analyses (Friston et al., 1997; Gitelman et al., 2003). In each subject, unadjusted activity was first extracted from volumes of interest [first eigenvariate of a spherical volume of interest (VOI); $3 \mathrm{~mm}$ radius] centered on the coordinates of the left and right hemisphere PPA. Each PPI regressor was then calculated (using the SPM5 utility) as the volume-by-volume product of the deconvolved VOI time series and a binary vector coding for condition blocks of the task design; -1 for the negative VS condition, +1 for the positive VS condition. The PPI was restricted to el blocks of the positive and negative VS conditions to prevent contamination by repetition reduction effects. For each participant, the PPI, condition coding, and VOI 
regressors were then separately reconvolved with the canonical HRF and modeled with the same specifications as the above first-level SPMs. In this way, a significant effect for PPI was not attributable to any main effects of task, or, any condition-independent covariance. Two contrasts were specified for the interaction column $(1$ and -1$)$, reflecting activations either positively or negatively related to the PPI interaction term, respectively. Inference of statistical significance was determined using a $p_{\text {unc }}<$ 0.001 and voxel extent of 5 as base thresholds.

Whole-brain analysis. To examine differential effects of AI, positive effect $t$ contrasts for each valence condition (positive, neutral, negative) were examined at the second level using a repeated-measure ANOVA. Group-level $t$ contrasts used a height of $p_{\text {unc }}<0.001$ and voxel extent of 5 as base thresholds.

\section{Results}

\section{Behavioral measures}

AI

Self-report measures of affect induction were collected both during and after the fMRI task. Repeated-measures ANOVA of valence ratings demonstrated a significant differential effect of $\mathrm{AI}\left(F_{(2,28)}=53.36\right.$, $p<0.001)$. Positive ratings were significantly higher than neutral $\left(t_{(14)}=6.49\right.$, $p<0.001$ ), while neutral ratings were significantly higher than negative $\left(t_{(14)}=\right.$ 7.56, $p<0.001)$. Repeated-measures ANOVA of arousal ratings demonstrated a valence-independent effect of arousal relative to neutral $\left(F_{(2,28)}=3.65, p<0.001\right)$. Arousal ratings did not significantly differentiate positive and negative states $\left(t_{(14)}=\right.$ 0.66, $p=0.52$ ).

\section{VS task performance}

To examine whether our valence state induction differentiated performance on the visuospatial gender task, two separate repeated-measures ANOVAs were calculated to examine response latency and accuracy for gender judgment of the central face stimulus. For response latency, the 3 (valence) $\times 2$ (repetition) ANOVA failed to detect any significant differential effects, with an average response latency of $715 \mathrm{~ms}$ for judging the gender of the central face.

Response latencies by valence condition were as follows: positive $=710.1 \pm 29.4 \mathrm{~ms}$; neutral $=715.7 \pm 29.8 \mathrm{~ms}$; negative $=$ $718.0 \pm 29.6 \mathrm{~ms}$. For accuracy, mean performance (regardless of condition) was $82 \%$, consistent with moderate attentional demand tasks using rapid stimulus presentation (Yi et al., 2004). There was a main effect of valence $\left(F_{(2,30)}=3.94, p=0.03\right)$, demonstrating overall more accurate responses following positive AI. Accuracy by valence condition was as follows: positive $=$ $84.1 \% \pm 2.2$; neutral $=81.4 \% \pm 2.8$; negative $=81.1 \% \pm 4.0$. However, follow up contrasts did not reveal a reliable effect of positive relative to either neutral $\left(t_{(14)}=1.32, p<0.21\right)$ or negative $\left(t_{(14)}=1.70, p<0.11\right)$ conditions. There were no other significant main effects or interactions. Collectively, these reLeft; R, right.

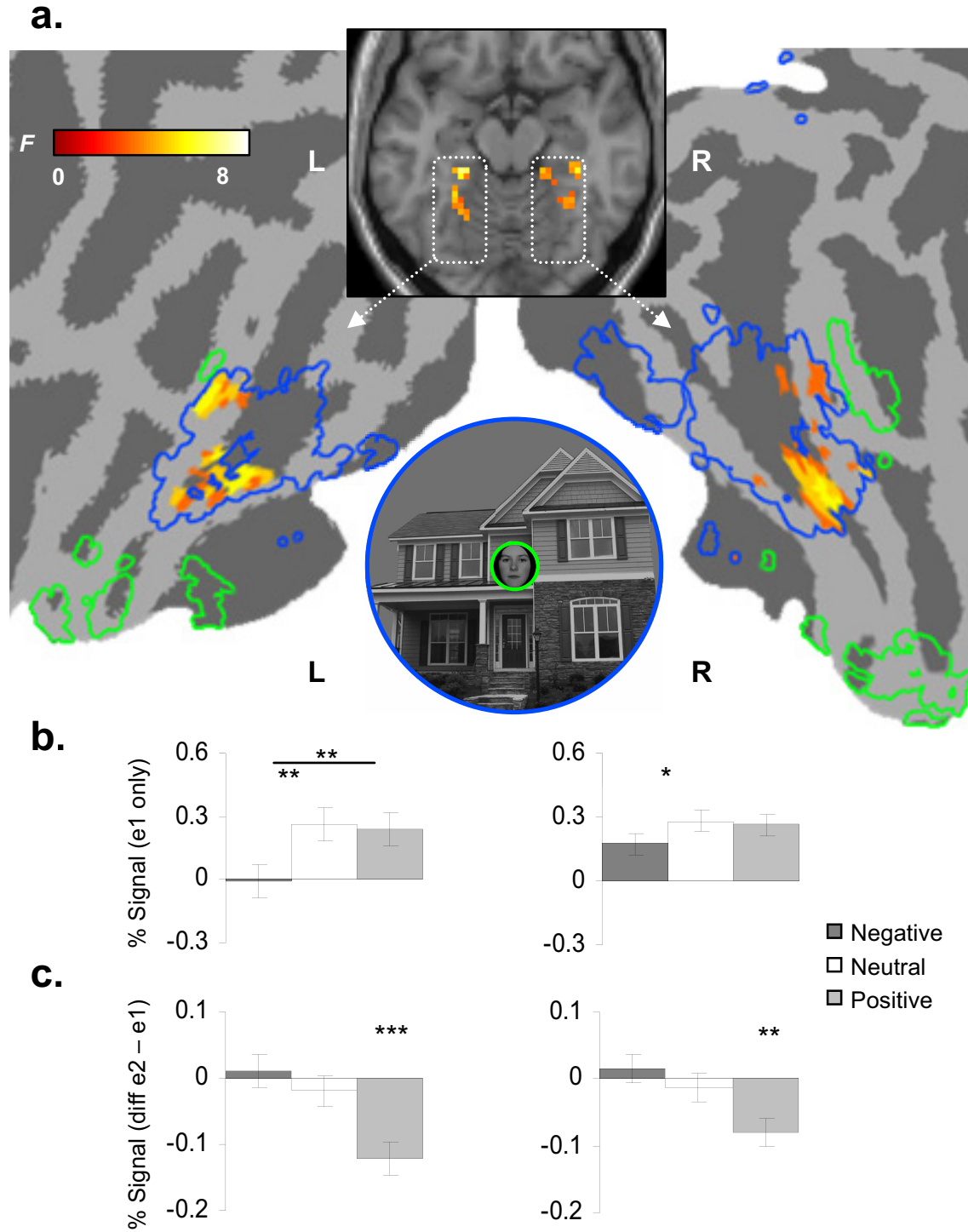

Figure 2. $\quad \boldsymbol{a}$, A significant valence $\times$ repetition interaction was found during the VS task bilaterally within the left and right PPA. Activation is superimposed on the template structural underlay (Colin27) (MNI axial $z$-coordinate $=-15$ ) and displayed on a cortical flat-map of the same template. For reference, PPA (dashed blue) and FFA (dashed green) ROls are also traced on the flat-map. $\boldsymbol{b}$, Novel place exposures (e1 blocks). $y$-axes, Percentage signal change at e1 blocks. In the left PPA, positive and neutra coliged significantly greater BOLD response to novel places than negative $\left({ }^{*} p<0.05 ;{ }^{* *} p<0.01 ;{ }^{* * *} p<0.001\right)$. Error bars reflect within-subject SEMs. c, Repetition reduction [e2 minus e1 blocks]. The valence $\times$ repetition interaction was values $=$ repetition reduction). Error bars reflect within-subject SEMs for differences between e2 and e1 blocks across subjects. L,

sponse time and accuracy data suggest there was no evidence of an AI-mediated trade-off in attention during the VS task.

\section{fMRI measures}

We examined how induction of positive and negative states influenced encoding of the place surround. The 3 (valence) $\times 2$ (repetition) repeated-measures ANOVA detected significant factorial interactions within the functionally defined PPA ROI, but not the FFA (Fig. 2a). We first decomposed the repetition factor of the full factorial model to examine the effect of valence on processing of novel place information, i.e., el blocks only. The three-level repeated-measures ANOVA detected a significant main effect of valence in the left PPA $\left(F_{(2,26)}=7.59, p=0.003\right)$. Within-subjects $t$ tests examining the valence factor revealed that 
a.

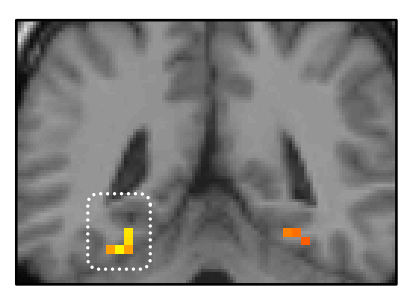

b.

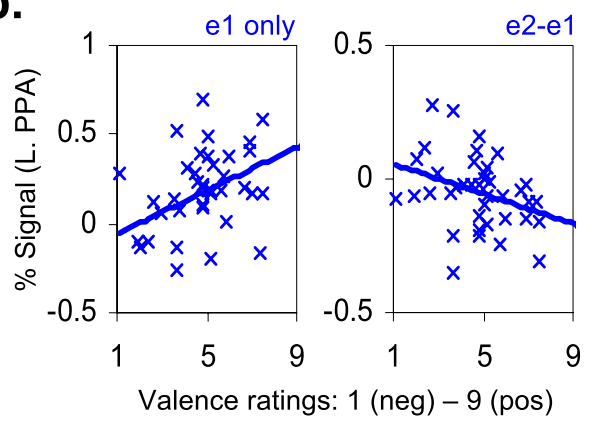

Figure 3. Extrastriate brain-behavior correlations. $\boldsymbol{a}$, The left PPA cluster (coronal slice: $y=-45$ ) is superimposed atop the template structural underlay (Colin27) and in MNI space. $\boldsymbol{b}$, Valence rating correlations with left PPA activity. A significant positive relationship was found for the magnitude of initial PPA response to novel places ( $y$-axis: percentage signal change at e1 blocks). A significant negative relationship was also found between behavioral ratings of valence and repetition reduction ( $y$-axis: difference between percentage signal change at second exposure and first exposure blocks). L., Left.

negative affect evoked significantly less response than both positive $\left(t_{\text {pos-neg(26) }}=3.27, p=0.003\right)$ and neutral $\left(t_{\text {neu-neg(26) }}=\right.$ $3.50, p=0.002$ ) conditions (Fig. $2 b$ ), consistent with decreased place encoding. The right PPA exhibited a comparable trend toward significance $\left(F_{(2,26)}=2.27, p=0.07\right)$; within-subjects $t$ tests confirmed a reduction in left PPA signal during negative affect $\left(t_{\text {pos-neg(26) }}=1.84, p=0.08 ; t_{\text {neu }- \text { neg(26) }}=2.25, p=0.03\right)$.

We next interrogated the valence $X$ repetition interaction detected by the full factorial model. Extracted signal from these clusters confirmed a significant valence $\times$ repetition interaction in both the left $\left(F_{(2,26)}=7.51, p=0.003 ; x=-30, y=-45, z=\right.$ $-15)$ and right $\left(F_{(2,26)}=5.76, p=0.008 ; x=36, y=-30, z=\right.$ -18 ) hemispheres. Higher magnitudes of repetition reduction were detected for positive affect compared with both neutral and negative conditions (Fig. $2 c$ ). Within-subjects $t$ tests examining repetition within each valence condition confirmed that both the right and left PPA interaction effects were selectively driven by positive affect (left PPA cluster: $t_{\text {pos e1-e2(26) }}=5.08, p=0.00003$, $t_{\text {neu e1-e2(26) }}<1, t_{\text {neg e1-e2(26) }}<1$; right PPA cluster: $t_{\text {pos e1-e2(26) }}$ $\left.=3.34, p=0.002, t_{\text {neu e } 1-\mathrm{e} 2(26)}<1, t_{\text {neg el }-\mathrm{e} 2(26)}<1\right)$, consistent with enhanced encoding of repeated places. In sum, during negative affective states, we observed a decrease in the magnitude of PPA response to novel places, and consequently, negligible repetition reduction. In contrast, our index of specific place encoding-reduced PPA response to repeated places-was only observed during positive affective states. Neutral states were characterized by an intermediate pattern, greater PPA BOLD response but negligible repetition reduction.

To ensure that valence and interaction effects detected during the VS task did not reflect incidental carryover effects from the preceding AI blocks, we also compared the effect of valence in the above PPA clusters during the AI conditions. No significant effects of valence were detected in either hemisphere (left: $F_{(2,26)}=$ 2.10, $p=0.14$; right: $F<1$ ). In addition, enhanced extrafoveal place encoding following positive affect induction blocks may indicate a trade-off between attention to the central task (faces) and to the surround (places). To address whether there was neural evidence of a volitional or reflexive trade-off between attention to the foveal face and the extrafoveal place surround, we examined whether there were valence related changes in face processing, as indexed by the localizer defined FFA ROI. There was a significant effect of valence in the FFA $\left(F_{(2,26)}=8.05, p=0.002\right.$; $x=39, y=-48, z=-18)$. In contrast with an attentional trade-off, positive affect evoked significantly greater signal com- pared with both neutral $\left(t_{(13)}=3.44, p=\right.$ $0.004)$ and negative $\left(t_{(13)}=3.21, p=\right.$ $0.007)$ conditions, consistent with an overall enhancement of cortical response during positive states.

\section{Brain-behavior relationships}

We next examined whether individual differences in self-reported affective valence correlated with altered cortical encoding of extrafoveal events. To do so, we examined the relationship between self-report measures of valence and magnitude of initial PPA response to novel place surrounds. A significant positive correlation for valence was detected for the left PPA cluster $(r=0.38, p=0.02)$ (Fig. $3 a$ ), whereby reports of increasing affective positivity corresponded with increased processing of novel places (Fig. $3 b$ ). We next examined whether the same self-report measures correlated with magnitude of PPA repetition reduction. This also revealed a significant negative correlation between reports of increasing positivity and magnitude of left PPA repetition reduction $(r=$ $-0.33, p=0.04$ ) (Fig. 3b). Thus, consistent with our factorial findings, individual variability in self-reported positive versus negative states predicted opposing effects on encoding of events in the extrafoveal visual field.

To address whether there was evidence of a trade-off between attention to the central foveal face and the extrafoveal place, we also examined whether self-reported valence correlated with FFA response. In contrast to our findings in the PPA, the valence regressor failed to identify a significant correlation with FFA percentage signal change at either first exposures $(r=0.11, p=0.52)$ or with repetition, i.e., e2 minus e1 $(r=0.11, p=0.50)$. Altered encoding of the unattended surround was therefore not associated with altered cortical response to the central attended face. In combination, these brain-behavior relationships between self reported affective state and our physiological metrics of perceptual encoding of place stimuli in the surround-magnitude of PPA response to novel and repeated places-indicates that positive affective states increase and negative states decrease encoding of extrafoveal events.

\section{Psychophysiological interaction analysis}

We next examined whether the influence of affective states involved differential engagement of the coupling between extrastriate and earlier visual cortices. To do so, the left and right PPA clusters reported in the preceding sections were entered as seed regions into separate PPI analyses, where the psychological variable of interest was positive or negative states during the first exposure of the VS task (e1 blocks). This enabled data-led interrogation as to whether positive and negative states differentially modulated functional covariance between the PPA and distal brain regions. The PPI originating from both the left and right PPA seeds revealed significant and highly selective activation bilaterally along the calcarine sulcus, consistent with extrafoveal regions of putative primary visual cortex (PVC) (Dougherty et al., 2003) (Fig. 4a,c). The PVC global maximum identified in each PPI was within the same hemisphere of the respective left and right PPA seed origin (left PVC, MNI coordinates: $x=-15, y=$ $-72, z=3$, Brodmann area 18; right PVC, $x=6, y=-78, z=15$, BA 18/17). A significant PPI therefore indicated that the func- 
a.
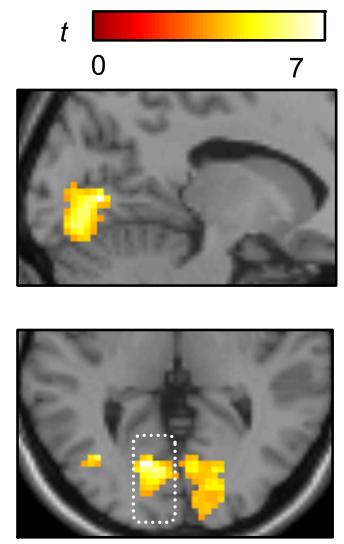

C.
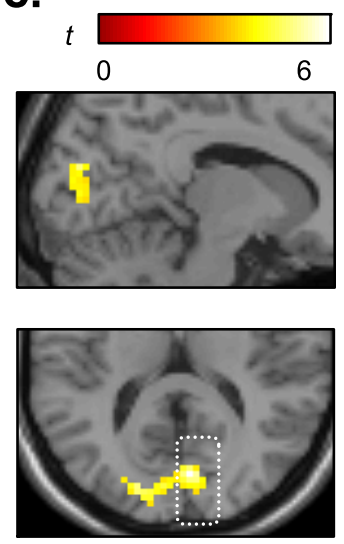

b.
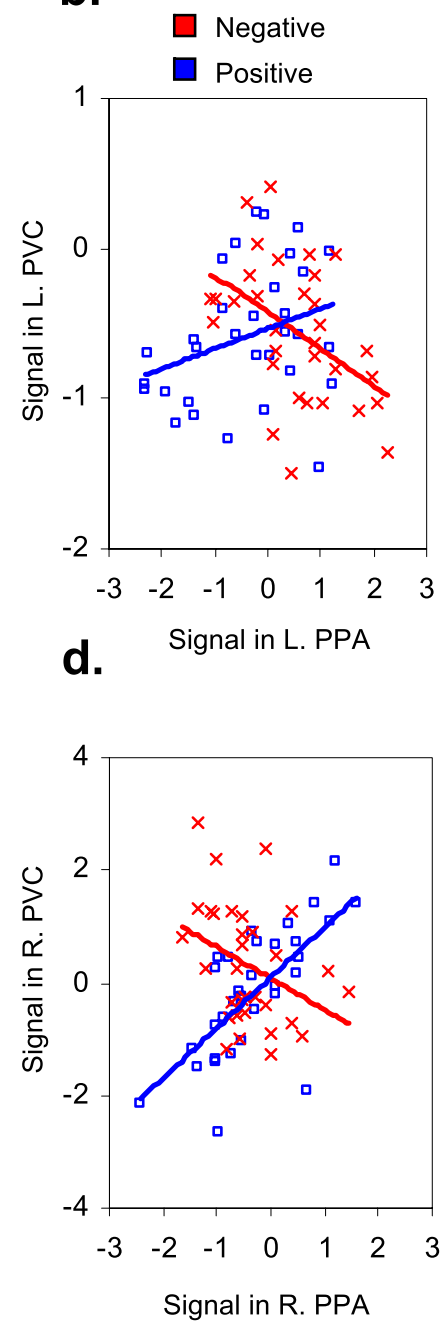

Figure 4. PPI analyses of valence-specific coupling with the PPA. $\boldsymbol{a}, \boldsymbol{c}$, The PPIs demonstrate that positive and negative states differentially modulated the covariance between the PPA and PVC bilaterally. Activation is superimposed on the template structural underlay (Colin27) (left: MNI sagittal $x$-coordinate $=-15$; axial $z$-coordinate $=3$; right: $x=6 ; z=15) \boldsymbol{b}, \boldsymbol{d}$, In both PPI seeds, positive affect (blue) elicited a positive coupling between PPA and PVC $\left(r_{\text {left }}=0.32\right.$, $\left.r_{\text {right }}=0.69\right)$, whereas negative affect (red) elicited a negative coupling $\left(r_{\text {left }}=-0.46, r_{\text {right }}\right.$ $=-0.36$ ). $x$-axes, Mean-corrected signal from the PPI term (the product of the respective PPA time series and condition regressors, averaged over 14 subjects). $y$-axes, Mean corrected signal from the respective PVC cluster maxima (first principal component of a $3 \mathrm{~mm}$ radius VOl, averaged over 14 subjects). The first two time points of each block were removed to account for the lag to peak BOLD response. L., Left; R., right.

tional relationship between the PVC and PPA differed significantly between positive and negative states, above and beyond any main effects of task as well as condition-independent covariance.

To identify the specific pattern of PVC-PPA covariance during positive and negative affect, we superimposed these correlations as separate series on the same scatter plot (Fig. $4 b, d$ ). The pattern of condition-specific functional covariance was highly symmetrical in both hemispheres. Positive affect elicited a positive coupling between PPA and PVC, whereas negative affect elicited a negative coupling between PPA and PVC, suggesting that common increases in PVC BOLD response may yield different and even opposing efferent modulation of extrastriate cortex under different affective states. Hence, positive affect appears to enhance and negative affect may diminish propagation of place information located in the extrafoveal surround.

\section{AI}

To investigate neural indices during and following affect induction, we first isolated the overall effect of emotional arousal (positive and negative) relative to neutral control. Positive and negative affect induction yielded significant activations across a distributed subcortical-brainstem-visual cortical system, consistent with research indicating enhanced visual processing of emotionally arousing images. Direct comparisons between positive and negative AI conditions also yielded significant differences in BOLD response. The [positive $>$ negative] $t$ contrast principally revealed activations in the orbitomedial (MNI coordinates: $x=18, y=48, z=-15$; BA 10) and ventromedial $(x=15, y=51, z=12$; BA 11) prefrontal cortices. The [positive $<$ negative] $t$ contrast yielded activation solely in the right amygdala $(x=21, y=0, z=-18)$. For AI summary statistics, see supplemental Table 1 (available at www.jneurosci.org as supplemental material).

We next examined whether these corticolimbic structures exhibited a sustained response during blocks of the interleaved VS task. To do so, we performed conjunction analyses between AI and VS tasks during positive [i.e., positive $>$ negative] and negative [i.e., positive $<$ negative] states. The conjunction therefore tested common state-specific activation at the voxel level. Inference of significance was assessed at a conjoint $\alpha$ level of $p_{\text {unc }}<$ 0.001 , and cluster extent of 5 voxels. During positive affect states, convergent activations were detected in the above mesocortical prefrontal loci, consistent with BA10/11 (Fig. 5a). An additional activation was detected more dorsally in the $\operatorname{mPFC}(x=18, y=$ $33, z=36$ ). During negative affect states, convergent activation was detected solely in the right amygdala locus (Fig. 5c). Together, the conjunction analyses provide physiological evidence for induction of a valence specific neural state in the absence of external affective cues, with mesocortical prefrontal and subcortical amygdalar regions exhibited sustained responses to positive and negative affect induction, respectively.

\section{Brain-behavior relationships}

In addition to the categorical modeling of AI, we examined whether continuous measures of self-reported affective valence correlated with BOLD percentage signal change in the mesocortical prefrontal and amygdalar loci (both the self-report measures and signal extractions were acquired using the same method as with the extrastriate ROIs). Significant positive correlations between self-reported positive valence with percentage signal change were found in the medial orbitofrontal cortex (mOFC) for both $\mathrm{AI}(r=0.44, p=0.006)$ and the interleaved VS ( $r=0.38$, $p=0.02$ ) tasks (Fig. $5 b$ ). In contrast, a significant negative correlation was observed between self-reported positive valence and amygdalar response for AI $(r=-0.33, p=0.04)$, while a comparable trend was observed during VS $(r=-0.29, p=0.08)$ (Fig. $5 d$ ). These brain-behavior relationships indicate that individual differences in positive and negative experiential states predicted opposing patterns of BOLD response in the mOFC and right amygdala, respectively.

\section{Discussion}

This study examined whether corticosubcortical pathways underlying positive and negatively valenced states differentially bias visual encoding in the extrastriate cortices. Positive affect increased and negative affect decreased the encoding of unattended peripheral information, as indexed by object-sensitive extrastri- 
a.

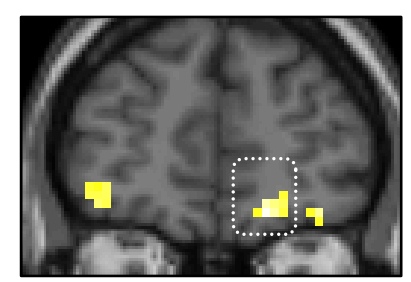

c.

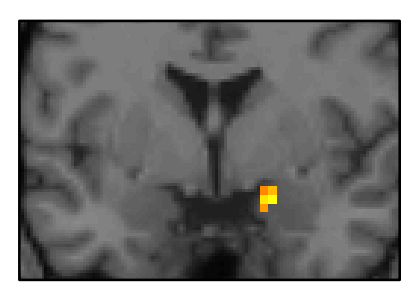

b.

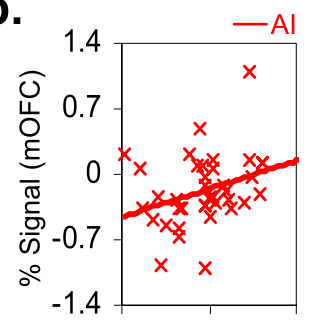

d.

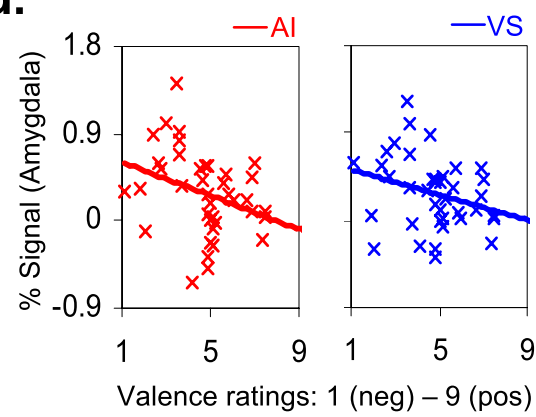

Figure 5. Corticosubcortical brain- behavior correlations a, c, Conjunction analyses between Al and VS tasks. Positive affect yielded activation in a right medial orbitofrontal locus (coronal slice: $y=48$ ). Negative affect revealed activation in the right amygdala $(y=0) \cdot \boldsymbol{b}, \boldsymbol{d}$, Brain-behavior correlations. A significant positive relationship was found between increasingly positive self-report ratings of valence and magnitude of BOLD response ( $y$-axes: percentage signal change) in the mOFC during both AI (red distribution) and VS (blue); $x$-axes (all panels): valence Likert scale 1 [negative (neg)] to 9 [positive (pos)]. Increasingly negative self-report ratings of valence correlated with increasing magnitude of BOLD response ( $Y$-axes: percentage signal change) in the amygdala during Al.

ate cortical responses in the PPA. These valence-dependent interactions with inferior temporal visual cortices observed during our visuospatial task occurred in the absence of exogenous affective cues, and, despite the fact that both the cognitive and perceptual demands of all visuospatial blocks were equated. Thus, in conjunction with existing behavioral evidence (Rowe et al., 2007), positive and negative affect appear to exert opposing influences on perceptual encoding, with positive states broadening and negative states narrowing one's FOV.

In the center/surround VS task, we inferred that modulation of the PPA reflects perceptual encoding of the unattended place surround (Tong et al., 1998; Yi et al., 2004). Consistent with our hypothesis that negative affect would narrow FOV, we observed a significant attenuation of PPA response to novel places during the negative condition. However, low magnitude of BOLD response does not necessarily equate with diminished processing. Adaptation to repeated stimuli has been detected in object selective extrastriate cortex despite negligible BOLD response to the first exposure (Avidan et al., 2002). Despite this possibility, we found that low BOLD signal in the PPA during negative states was also associated with abolished place adaptation, suggesting a genuine reduction in extrafoveal place processing. Although PPA response to novel places failed to differentiate the neutral and positive conditions, only positive affect yielded repetition reduction in the PPA. Neutral states were thus characterized by an intermediate pattern - greater initial PPA response than negative states but negligible repetition reduction. Consistent with this observation, our brain-behavior analyses revealed a linear relationship between continuous self-report measures of valence and both the magnitude of PPA response to novel places and repetition reduction to repeated places. Our continuous (i.e., selfreport) data might therefore reflect a greater degree of sensitivity to valence-dependent changes in extrastriate processing.
Several neuroimaging studies have provided evidence challenging the object selectivity of the FFA and PPA to "optimal" stimulus categories - faces and places, respectively (Avidan et al., 2002; GrillSpector et al., 2006). In particular, Avidan et al. (2002) detected neural adaptation to places in the FFA and adaptation to faces in the PPA, indicating a degree of nonmodularity in these extrastriate regions. The present study design obviates this potential confound because only place information was repeated. Given that faces were always novel throughout our experiment, we would therefore not expect face selective neurons in the PPA to influence our valence-dependent adaptation effects. As such, our experimental design precludes several alternative explanations for the valence effects observed in the extrastriate cortex.

Notably, repetition-related BOLD reductions in the inferior temporal cortices have been found to correlate with altered behavioral performance, indicating a potential origin in later response selection, rather than earlier perceptual encoding (Turk-Browne et al., 2006). However, repetition-related improvements in behavioral performance have been fully dissociated from place-selective BOLD response in the PPA, with the magnitude of PPA attenuation to repeated places unaffected by changes in task demands and task performance (Xu et al., 2007). In fact, repetition-related reductions in the PPA appear to be more influenced by bottom-up rather than top-down modes of attentional processing (Yi et al., 2004). In combination, these results suggest that the present finding of an interaction between the valence of internal affective states and repetition reduction related to unattended stimulus events likely originates in earlier perceptual encoding rather than later information processing stages.

The extrastriate PPA findings represent a relatively late nonretinotopically organized stage of visual selection, and thus do not demonstrate that affective influences extend to the earliest stages of visual cortical processing. To better appreciate the cortical pathways involved in gating PPA response, we additionally used PPI analyses to interrogate how positive and negative states differentially modulated functional covariance between the PPA and distal neuronal populations. The PPI analyses demonstrated that affective valence modulated the relationship between early stages of visual selection in a region along the calcarine sulcus, consistent with extrafoveal representations of PVC and later stages of object encoding in the inferior temporal cortex (PPA). Positive and negative affect modulated this PVC-PPA functional covariance in an opposing manner. Specifically, positive affect may have enhanced the propagation of place-sensitive processing, whereby increasing PVC activation predicted increasing PPA activation. In contrast, negative affect may have reflected early gating of this propagation of place information, whereby increasing PVC activity predicted reduced PPA response.

The PPI results therefore indicate that affective states modulate perceptual encoding via altered coupling with posterior sensory cortices as opposed to a more indirect top-down influence via altered engagement of frontal-parietal attentional networks. 
Rather than engaging attention more or less, positive and negative states may have a more direct influence on the processing dynamics in primary, secondary, and higher associative cortices (Anderson and Phelps, 2001; Pessoa and Ungerleider, 2004; Anderson, 2005; Dolan, 2007). A better understanding of these putative valence-specific processing modes may be gleaned from brain regions recruited during positive and negative states. Valence-specific patterns of BOLD response were found in the amygdala and mOFC during negative and positive affect induction, respectively, which were also sustained during the interleaved visuospatial task, when external affective cues were absent altogether. Additionally, self-reported valence ratings correlated inversely with the amygdala and mOFC as well as with extrafoveal place processing indexed by the PPA. Thus, activity in these affective substrates may represent neural states that differentially bias visual cortical sensitivity to perceptual inputs.

Convergent neuroimaging evidence suggests that under many conditions, the amygdala is equally responsive during both positive and negative affect. Comparable amygdalar responses have been observed during exposure to positive and negative facial expressions (Breiter et al., 1996; Kim et al., 2003), episodic recall of positive and negative scenes (Hamann et al., 1999), as well as exposure to aversive and pleasant odors (Anderson et al., 2003; Winston et al., 2005) and tastes (Small et al., 2003). A recent meta-analysis examining several hundred imaging studies in affective neuroscience corroborates this valence-independent (relative to neutral) but arousal-dependent (relative to neutral) sensitivity profile of amygdalar response (Costafreda et al., 2008). Consistent with this body of work, we found that positive and negative stimuli evoked greater bilateral amygdalar response compared with neutral. However, we also observed greater right amygdala activation during negative affect induction and the interleaved visuospatial task compared with the respective positive affect conditions, despite equivalent self-reported arousal levels in both valence conditions. How might these valenceindependent and -dependent amygdalar findings be reconciled? One possible account comes from research suggesting that the amygdala is sensitive to more generalized sources of arousal, such as predictive uncertainty about the environment (Davis and Whalen, 2001; Kim et al., 2003) or motivational relevance with respect to one's current goals (Cunningham et al., 2008; Van Bavel et al., 2008). These sources of arousal yield amygdalar activation in both positive and negative contexts, but with enhanced activity observed in the negative (Kim et al., 2003; Cunningham et al., 2008). This negativity bias may represent a sensory adaptive function, enhancing not the affective processing of negative stimuli, but rather a top-down optimization for sensory processing of negative stimuli. For instance, the amygdala has been shown to support vigilance toward negatively arousing relative to other sensory events competing for processing resources (Anderson and Phelps, 2001; Hurlemann et al., 2007), and, increased selectivity (i.e., tuning) of the sensory cortices following fear conditioning (Morris et al., 1998). Further, amygdala and extrastriate responses correlate during negative image processing (Sabatinelli et al., 2005; Kensinger et al., 2007b), hypothetically biasing a more circumscribed selective propagation of sensory information from posterior perceptual cortices (Kensinger et al., 2007b). Rather than attributing our valence-dependent amygdala finding to the emergence of a negative affective state, we propose instead that the amygdala may facilitate a specific mode of sensory information processing with a narrow FOV as a perceptual correlate, triggered by negative and possibly also by high-arousal positive states (Kim et al., 2003; Gable and Harmon-Jones, 2008).
Neural theories on the role of positive affect in cognition highlight the potential influence of mesocortical dopamine systems on inhibitory and cognitive control (Dreisbach, 2006), which is thought to facilitate switches in attentional set and perspective, and more generally, override dominant modes of processing (Ashby et al., 1999). For example, dopamine agonists disrupt latent inhibition, a measure of attentional inhibition of irrelevant stimuli (Swerdlow et al., 2003). However, in our task, positive affect did not engage mesolimbic dopaminergic circuits associated with appetitive-approach motivation (Breiter et al., 1997; Aharon et al., 2001; Knutson et al., 2001) and more exploitative forms of incentive learning (McClure et al., 2003; O’Doherty et al., 2004; Daw et al., 2006). In contrast, we found that self-reported positive affect correlated with sustained activation of a mesocortical target region in the mOFC, a region in which reward-related processing may extend to involve more exploratory modes of thought and behavior, such as suboptimal exploratory selections in an incentive learning gambling task (Daw et al., 2006), improvisation (Limb and Braun, 2008), and insightful problem solving (Subramaniam et al., 2009). This neuroimaging evidence complements a long line of behavioral research linking positive emotion with increased scope and flexibility of cognition (Isen et al., 1987; Ashby et al., 1999; Fredrickson, 2004; Rowe et al., 2007). The present findings suggest these influences may extend beyond high-level cognition to earlier stages of perceptual processing, resulting in a more exploratory and global analysis of perceptual inputs.

Collectively, the present data provide evidence that internal affective states alter visual cortical responses in a valencedependent manner, with positive states increasing and negative states decreasing the scope of perceptual encoding. The origins of these opposing valence-dependent influences may arise from more primitive opposing action tendencies supported by subcortical structures, such as the amygdala, to enhance stereotyped reflexes (Davis and Whalen, 2001) and mesocortical prefrontal regions that enhance novel or exploratory appetitive behavior (Daw et al., 2006). We hypothesize that activation of neural states originating from these systems may represent fundamental opposing biases on information processing ranging from thought to perception and action.

\section{References}

Aguirre GK, Zarahn E, D’Esposito M (1998) An area within human ventral cortex sensitive to "building" stimuli: evidence and implications. Neuron 21:373-383.

Aharon I, Etcoff N, Ariely D, Chabris CF, O'Connor E, Breiter HC (2001) Beautiful faces have variable reward value: $\mathrm{fMRI}$ and behavioral evidence. Neuron 32:537-551.

Anderson AK (2005) Affective influences on the attentional dynamics supporting awareness. J Exp Psychol Gen 134:258-281.

Anderson AK, Phelps EA (2001) Lesions of the human amygdala impair enhanced perception of emotionally salient events. Nature 411:305-309.

Anderson AK, Christoff K, Stappen I, Panitz D, Ghahremani DG, Glover G, Gabrieli JD, Sobel N (2003) Dissociated neural representations of intensity and valence in human olfaction. Nat Neurosci 6:196-202.

Ashby FG, Isen AM, Turken AU (1999) A neuropsychological theory of positive affect and its influence on cognition. Psychol Rev 106:529-550.

Avidan G, Hasson U, Hendler T, Zohary E, Malach R (2002) Analysis of the neuronal selectivity underlying low fMRI signals. Curr Biol 12:964-972.

Bradley MM, Sabatinelli D, Lang PJ, Fitzsimmons JR, King W, Desai P (2003) Activation of the visual cortex in motivated attention. Behav Neurosci 117:369-380.

Breiter HC, Etcoff NL, Whalen PJ, Kennedy WA, Rauch SL, Buckner RL, Strauss MM, Hyman SE, Rosen BR (1996) Response and habituation of the human amygdala during visual processing of facial expression. Neuron 17:875-887.

Breiter HC, Gollub RL, Weisskoff RM, Kennedy DN, Makris N, Berke JD, 
Goodman JM, Kantor HL, Gastfriend DR, Riorden JP, Mathew RT, Rosen BR, Hyman SE (1997) Acute effects of cocaine on human brain activity and emotion. Neuron 19:591-611.

Brett M, Anton JL, Valabregue R, Poline JB (2002) Region of interest analysis using an SPM toolbox. Paper presented at Eighth International Conference on Functional Mapping of the Human Brain. Sendai, Japan, June.

Christianson SA (1992) Emotional stress and eyewitness memory: a critical review. Psychol Bull 112:284-309.

Cohen JD, McClure SM, Yu AJ (2007) Should I stay or should I go? How the human brain manages the trade-off between exploitation and exploration. Philos Trans R Soc Lond B Biol Sci 362:933-942.

Costafreda SG, Brammer MJ, David AS, Fu CH (2008) Predictors of amygdala activation during the processing of emotional stimuli: a metaanalysis of 385 PET and fMRI studies. Brain Res Rev 58:57-70.

Cunningham WA, Van Bavel JJ, Johnsen IR (2008) Affective flexibility: evaluative processing goals shape amygdala activity. Psychol Sci 19:152-160.

Davis M, Whalen PJ (2001) The amygdala: vigilance and emotion. Mol Psychiatry 6:13-34.

Daw ND, O'Doherty JP, Dayan P, Seymour B, Dolan RJ (2006) Cortical substrates for exploratory decisions in humans. Nature 441:876-879.

Derryberry D, Tucker DM (1994) Motivating the focus of attention. In: The heart's eye: emotional influences in perception and attention (Niedenthal PM, Kitayama S, eds), pp 167-196. San Diego: Academic.

Dolan RJ (2007) The human amygdala and orbital prefrontal cortex in behavioural regulation. Philos Trans R Soc Lond B Biol Sci 362:787-799.

Dougherty RF, Koch VM, Brewer AA, Fischer B, Modersitzki J, Wandell BA (2003) Visual field representations and locations of visual areas V1/2/3 in human visual cortex. J Vis 3:586-598.

Dreisbach G (2006) How positive affect modulates cognitive control: the costs and benefits of reduced maintenance capability. Brain Cogn 60:11-19.

Epstein R, Kanwisher N (1998) A cortical representation of the local visual environment. Nature 392:598-601.

Fenske MJ, Eastwood JD (2003) Modulation of focused attention by faces expressing emotion: evidence from flanker tasks. Emotion 3:327-343.

Fredrickson BL (2004) The broaden-and-build theory of positive emotions. Philos Trans R Soc Lond B Biol Sci 359:1367-1378.

Friston KJ, Jezzard P, Turner R (1994) Analysis of functional MRI timeseries. Hum Brain Mapp 1:153-171.

Friston KJ, Buechel C, Fink GR, Morris J, Rolls E, Dolan RJ (1997) Psychophysiological and modulatory interactions in neuroimaging. Neuroimage 6:218-229.

Gable PA, Harmon-Jones E (2008) Approach-motivated positive affect reduces breadth of attention. Psychol Sci 19:476-482.

Gasper K, Clore GL (2002) Attending to the big picture: mood and global versus local processing of visual information. Psychol Sci 13:34-40.

Genovese CR, Lazar NA, Nichols T (2002) Thresholding of statistical maps in functional neuroimaging using the false discovery rate. Neuroimage 15:870-878.

Gitelman DR, Penny WD, Ashburner J, Friston KJ (2003) Modeling regional and psychophysiologic interactions in fMRI: the importance of hemodynamic deconvolution. Neuroimage 19:200-207.

Goel V, Dolan RJ (2003) Reciprocal neural response within lateral and ventral medial prefrontal cortex during hot and cold reasoning. Neuroimage 20:2314-2321.

Grill-Spector K, Sayres R, Ress D (2006) High-resolution imaging reveals highly selective nonface clusters in the fusiform face area. Nat Neurosci 9:1177-1185.

Hamann SB, Ely TD, Grafton ST, Kilts CD (1999) Amygdala activity related to enhanced memory for pleasant and aversive stimuli. Nat Neurosci 2:289-293.

Hurlemann R, Matusch A, Hawellek B, Klingmuller D, Kolsch H, Maier W, Dolan RJ (2007) Emotion-induced retrograde amnesia varies as a function of noradrenergic-glucocorticoid activity. Psychopharmacology (Berl) 194:261-269.

Isen AM, Daubman KA, Nowicki GP (1987) Positive affect facilitates creative problem solving. J Pers Soc Psychol 52:1122-1131.

Kanwisher N, McDermott J, Chun MM (1997) The fusiform face area: a module in human extrastriate cortex specialized for face perception. J Neurosci 17:4302-4311.

Kensinger EA, Garoff-Eaton RJ, Schacter DL (2007a) Effects of emotion on memory specificity in young and older adults. J Gerontol B Psychol Sci Soc Sci 62:P208-215.

Kensinger EA, Garoff-Eaton RJ, Schacter DL (2007b) How negative emo- tion enhances the visual specificity of a memory. J Cogn Neurosci 19:1872-1887.

Kim H, Somerville LH, Johnstone T, Alexander AL, Whalen PJ (2003) Inverse amygdala and medial prefrontal cortex responses to surprised faces. Neuroreport 14:2317-2322.

Knutson B, Adams CM, Fong GW, Hommer D (2001) Anticipation of increasing monetary reward selectively recruits nucleus accumbens. J Neurosci 21:RC159.

Lang PJ, Bradley MM, Fitzsimmons JR, Cuthbert BN, Scott JD, Moulder B, Nangia V (1998) Emotional arousal and activation of the visual cortex: an fMRI analysis. Psychophysiology 35:199-210.

Lang PJ, Bradley MM, Cuthbert BN (1999) International affective picture system (IAPS): technical manual and affective ratings. Gainesville, FL: Gainesville University of Florida, Center for Research in Psychophysiology.

Levy I, Hasson U, Avidan G, Hendler T, Malach R (2001) Center-periphery organization of human object areas. Nat Neurosci 4:533-539.

Levy I, Hasson U, Harel M, Malach R (2004) Functional analysis of the periphery effect in human building related areas. Hum Brain Mapp 22:15-26.

Limb CJ, Braun AR (2008) Neural substrates of spontaneous musical performance: an FMRI study of jazz improvisation. PLoS ONE 3:e1679.

Macey PM, Macey KE, Kumar R, Harper RM (2004) A method for remova of global effects from fMRI time series. Neuroimage 22:360-366.

McClure SM, Berns GS, Montague PR (2003) Temporal prediction errors in a passive learning task activate human striatum. Neuron 38:339-346.

Morris JS, Friston KJ, Dolan RJ (1998) Experience-dependent modulation of tonotopic neural responses in human auditory cortex. Proc Biol Sci 265:649-657.

O’Doherty J, Dayan P, Schultz J, Deichmann R, Friston K, Dolan RJ (2004) Dissociable roles of ventral and dorsal striatum in instrumental conditioning. Science 304:452-454.

Pessoa L, Ungerleider LG (2004) Neuroimaging studies of attention and the processing of emotion-laden stimuli. Prog Brain Res 144:171-182.

Puce A, Allison T, Gore JC, McCarthy G (1995) Face-sensitive regions in human extrastriate cortex studied by functional MRI. J Neurophysiol 74:1192-1199.

Rovamo J, Virsu V, Näsänen R (1978) Cortical magnification factor predicts the photopic contrast sensitivity of peripheral vision. Nature 271:54-56.

Rowe G, Hirsh JB, Anderson AK (2007) Positive affect increases the breadth of attentional selection. Proc Natl Acad Sci U S A 104:383-388.

Sabatinelli D, Bradley MM, Fitzsimmons JR, Lang PJ (2005) Parallel amygdala and inferotemporal activation reflect emotional intensity and fear relevance. Neuroimage 24:1265-1270.

Small DM, Gregory MD, Mak YE, Gitelman D, Mesulam MM, Parrish T (2003) Dissociation of neural representation of intensity and affective valuation in human gustation. Neuron 39:701-711.

Subramaniam K, Kounios J, Parrish TB, Jung-Beeman M (2009) A brain mechanism for facilitation of insight by positive affect. J Cogn Neurosci 21:415-432.

Swerdlow NR, Stephany N, Wasserman LC, Talledo J, Sharp R, Auerbach PP (2003) Dopamine agonists disrupt visual latent inhibition in normal males using a within-subject paradigm. Psychopharmacology (Berl) 169:314-320.

Tong F, Nakayama K, Vaughan JT, Kanwisher N (1998) Binocular rivalry and visual awareness in human extrastriate cortex. Neuron 21:753-759.

Turk-Browne NB, Yi DJ, Chun MM (2006) Linking implicit and explicit memory: common encoding factors and shared representations. Neuron 49:917-927.

Van Bavel JJ, Packer DJ, Cunningham WA (2008) The neural substrates of in-group bias: a functional magnetic resonance imaging investigation. Psychol Sci 19:1131-1139.

Vuilleumier P (2005) How brains beware: neural mechanisms of emotional attention. Trends Cogn Sci 9:585-594.

Weymouth FW (1958) Visual sensory units and the minimal angle of resolution. Am J Ophthalmol 46:102-113.

Winston JS, Gottfried JA, Kilner JM, Dolan RJ (2005) Integrated neural representations of odor intensity and affective valence in human amygdala. J Neurosci 25:8903-8907.

Xu Y, Turk-Browne NB, Chun MM (2007) Dissociating task performance from fMRI repetition attenuation in ventral visual cortex. J Neurosci 27:5981-5985.

Yi DJ, Woodman GF, Widders D, Marois R, Chun MM (2004) Neural fate of ignored stimuli: dissociable effects of perceptual and working memory load. Nat Neurosci 7:992-996. 